\section{JTI}

JOURNAL OF

TRAUMA AND INJURY

\title{
Esophageal Rupture Due to Diving in Shallow Waters
}

\author{
Sung Ho Han, M.D. ${ }^{1}$, Soon-Ho Chon, M.D., Ph.D. ${ }^{2}$, Jong Hyun Lee ${ }^{2}$, \\ Min Koo Lee, M.D. ${ }^{1}$, Oh Sang Kwon, M.D. ${ }^{1}$, Kyoung Hwan Kim, M.D. ${ }^{1}$, \\ Jung Suk Kim, M.D. ${ }^{1}$, Ho hyoung Lee, M.D. ${ }^{1}$, June Raphael Chon, M.D. ${ }^{3}$ \\ Departments of ${ }^{1}$ Traumatology and ${ }^{2}$ Thoracic and Cardiovascular Surgery, Cheju Halla General \\ Hospital, Jeju, Korea \\ ${ }^{3}$ St. Johnsbury Academy, Jeju, Korea
}

Received: October 12, 2017

Revised: December 14, 2017

Accepted: December 26, 2017

\section{Correspondence to}

Soon-Ho Chon, M.D., Ph.D.

Department of Thoracic and Cardiovascu-

lar Surgery, Cheju Halla General Hospital,

65 Doryeong-ro, Jeju 63127, Korea

Tel: $+82-64-740-5039$

Fax: $+82-64-743-3110$

E-mail: sh.chon@hotmail.com
Delayed esophageal rupture due to blunt injury is not new. However, rupture due to suspected barotrauma is very rare. We describe a case of esophageal rupture in a male 24 -year-old patient after diving in shallow waters. The patient was quadriplegic and could not experience the typical chest pain related to rupture and resulting mediastinitis. The rupture was discovered 4 days after emergency decompressive laminectomy and fusion for his cervical spine. The rupture was evidently caused by barotrauma and was discovered four days after admission. He underwent primary closure and pericardial flap as a life-saving procedure.

Keywords: Esophageal perforation; Wounds and injuries; Barotrauma

\section{INTRODUCTION}

Esophageal rupture caused by blunt trauma has been reported to occur in $0.001 \%$ of all esophageal ruptures and they most commonly occur above the level of the carina [1]. Esophageal rupture caused distal to the carina and distal from the site of blunt injury due to barotrauma is an extremely rare event. Seventy percent of esophageal ruptures due to blunt injury are found late and have poor prognosis [1,2]. We report a rare case of distal esophageal rupture after blunt injury caused by barotrauma.

\section{CASE REPORT}

A 24-year-old male had visited our emergency center with symptoms of quadriplegia 
caused by diving in shallow waters under the influence of alcohol. The patient had just finished compulsory military service as required by his country. He worked as an electrician before his service. His initial neurologic examination presented absent motor and sensory responses below his fourth thoracic nerve distribution. He had exaggerated knee and ankle jerk reflexes. He had leukocytosis at 15,220. His c-spine simple anteroposterior and lateral views presented c-spine dislocation and his magnetic resonance image scans revealed dislocation and cord contusion. C4-5 traumatic spondylolisthesis grade 3 with intervertebral disc rupture, bilateral interfacet dislocation and severe compression of his spinal cord findings were seen on his magnetic resonance imaging. His initial chest tomographic scans revealed no abnormal findings. Initial diagnosis was c-spine dislocation and cervical cord injury. He thus underwent an emergency operation. Our neurosurgeon performed a posterior approach decompressive laminectomy and reduction and lateral mass fusion of his cervical 3rd, 4th, 5th, and 6th vertebrae. On postoperative 3rd day he was presenting signs of respiratory distress and was placed on a ventilator. On the 4 th postoperative day he developed bilateral pneumothorax with subcutaneous emphysema and had an emergency chest tube insertion. The chest tube drained a dark colored and malodorous fluid. The amylase level was 113,256 IU/L, lipase level 16 IU/L, with a red blood cell count at 56,000. This prompted studies for delayed esophageal rupture and he underwent gastrofiberoscopy. A repeat chest tomographic scan was taken and presented mediastinitis, pneumomediastinum and bilateral pneumothoraces (Figs. 1, 2). Results presented a $1 \mathrm{~cm}$ tear in his lower esophagus near his esophago-gastric junction (Fig. 3). Decision for emergent primary closure was done.

The operation was scheduled and the procedure was done with double lumen intubation. The patient was placed in the right down decubitus position with arm elevated and placed over an arm rest. A full anterolateral thoracotomy incision was done over his 7 th intercostal space. Debridement of infected tissue was done and the site of leakage identified. Primary continuous suture was done with antibiotic coated 3-0 vicryl sutures. Pericardium flap below his phrenic nerve was sutured over the suture line. Massive irrigation and a 24 Fr. chest tube was

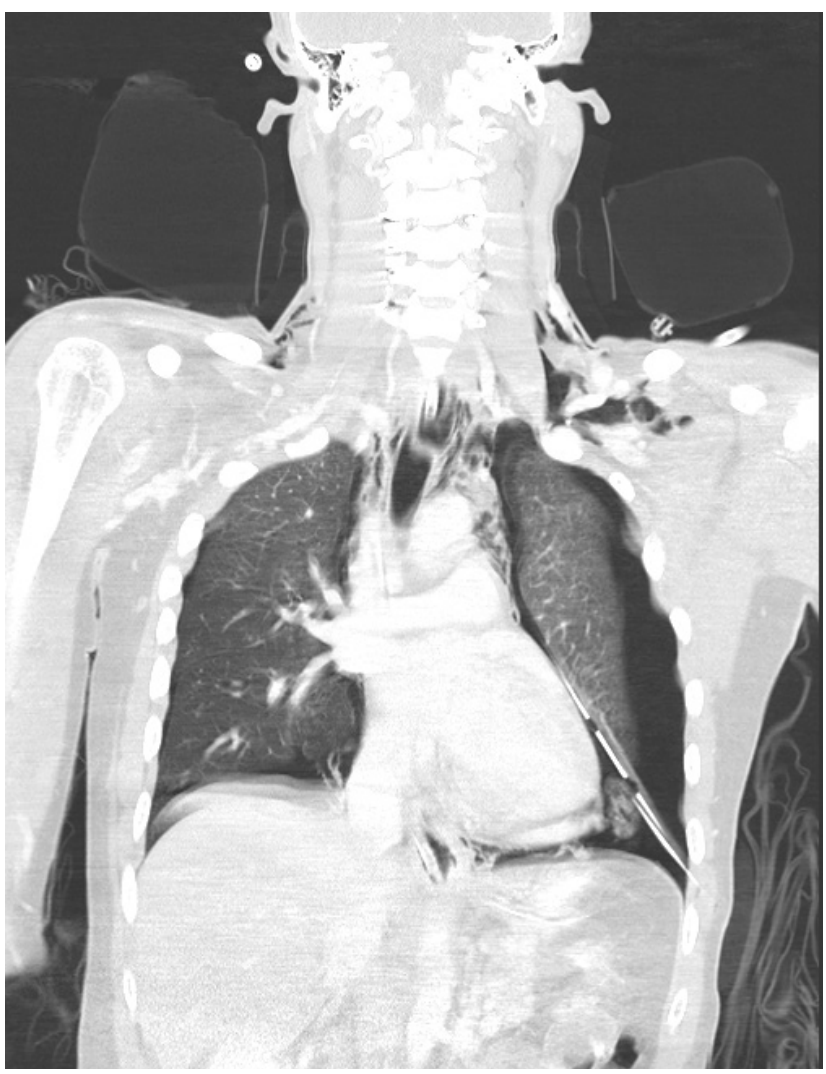

Fig. 1. Chest tomographic scan taken 4 days after blunt injury to the neck, showing pneumomediastinum and bilateral pneumothoraces after tube insertion.

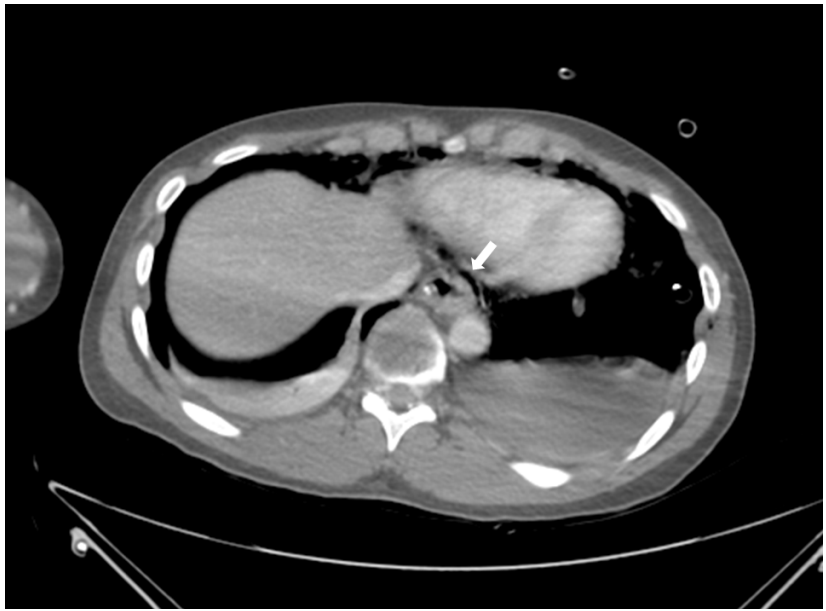

Fig. 2. Chest tomographic axial scan showing mediastinitis (arrow).

inserted in his mediastinum and a 28 Fr. chest tube was inserted in his pleural cavity.

On his postoperative 2 nd day, a tracheostomy was done. On his postoperative 5th day his pleural tube was 


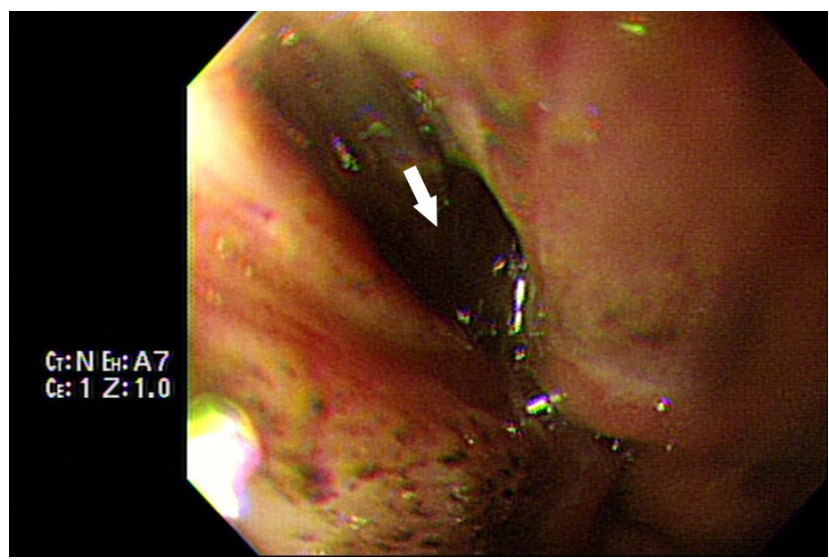

Fig. 3. The $1 \mathrm{~cm}$ tear (arrow) seen on a captured image during gastrofiberoscopy done 4 days after the neurosurgical procedure.

removed and on his 7th postoperative day an esophagogram was performed using gastrograffin. No leak was seen and thus, the mediastinal tube was removed and he was then retransferred to our neurosurgery department.

On his 92nd hospital day he was transferred to his place of residence. Unfortunately he was still quadriplegic, but he is alive.

\section{DISCUSSION}

The incidence of blunt esophageal rupture has been reported to be $0.0002 \%$ of all blunt chest injuries [1]. There have been reports of esophageal rupture in the cervical area due to fall down [3], however, esophageal rupture after neck injury after diving at a site far from the injury site is unaccounted for. The mechanism of injury cannot be explained by the site of injury itself. The patient has had no history of esophageal disease. Esophageal rupture caused by steroids has been reported in a patient with kidney transplantation [4]. High doses of immunosuppressive drugs and high doses of corticosteroids may cause ulceration and perforation. Other etiologic mechanisms may include barotrauma during impact. However rare, there has been one such reported case [2]. We believe the impact may have caused a surge of air through the esophagus, causing the barotrauma.

Manifestations of esophageal rupture include pain, vomiting, hematemesis, dysphagia, odynophagia, dysphonia, dyspnea, and/or fever [5]. In our particular case the most common symptom of pain was not possible to evaluate due to his quadriplegic condition. Other symptoms would have also been obscured by his condition.

The choice of management of esophageal rupture in this particular case was very difficult. Nonoperative management has a mortality of $20-38 \%$ and a surgical alternative in a patient with late diagnosis of over 24 hours is $27 \%$ [5]. In patients with esophageal rupture diagnosed more than 48 hours after the incident where there is significant mediastinitis, an esophageal diversion and gastrostomy operation would be indicated. In our particular case, we chose to perform primary closure with a pericardial flap. Fortunately, in our case, this was successful.

We report a case of esophageal rupture caused by barotrauma after neck injury in a patient who took a dive in shallow waters. Prompt diagnosis is necessary and awareness of the possibility of such an event distal to the site of injury is of upmost importance for survival.

\section{REFERENCES}

1. Cedeño A, Echeverría K, Vázquez J, Delgado A, Rodríguez-Ortiz P. Intrathoracic esophageal rupture distal to the carina after blunt chest trauma: case-report. Int J of Surg Case Rep 2015;16:184-6.

2. Muneer M, Abdelrahman H, El-Menyar A, Afifi I, Al-Hassani A, AlMadani A, et al. External air compression: a rare case of blunt esophageal injury, managed by a stent. Int J Surg Case Rep 2014;5:620-3.

3. van Heijl M, Saltzherr TP, van Berge Henegouwen MI, Goslings JC. Unique case of esophageal rupture after a fall from height. BMC Emerg Med 2009;9:24 .

4. Amini M, Ghorbani Abdehgah A, Molavi B, Radmard AR, Ali Askari A. Esophageal perforation in a patient with kidney transplantation. Iran J Kidney Dis 2016;10:233-6.

5. Kaman L, Iqbal J, Kundil B, Kochhar R. Management of esophageal perforation in adults. Gastroenterology Res 2010;3:235-44. 